\title{
Livestock Detection and Counting Using CNN
}

\section{Harsha M V ${ }^{1}$, Karthik J Bharadwaj ${ }^{2}$ and Lohith Raj S N ${ }^{3}$}

\author{
${ }^{1,2,3}$ Computer Science \& Engineering, Bangalore Institute of Technology \\ Bangalore, Karnataka - 560004, India
}

\begin{abstract}
Livestock sector is considered one of the major sector. In both the international economy and socialeconomic development of the countries it plays a very crucial role.Efficient and reliable detection of livestock animals in the local ranch is essential. This project develops an algorithm to detect the animals and to count them. Since there are a large number of animals personally identifying and counting them will be a very difficult task. This application classifies livestock animals based on the pictures fed by live camera so we can monitor and keep count of them more correctly. Livestock Animal detection and identification can help to notify intruder. This will be achieved by applying effective deep learning $\mathrm{CNN}$ algorithm. Here we are using Convolutional Neural network(CNN) to identify the type of livestock animal. BLOB is used to store information in the database.
\end{abstract}

Keywords: CNN algorithm, BLOB, Convolutional Neural Network.

\section{Introduction}

Livestock subsector has large contribution to developing countries and local economy, especially in the provision of food and dairy products for the growing human population, supply of raw materials to the growing industrial sector and about $42 \%$ of the global GDP and serves as the quickest growing agricultural market and a major contributor to food and as well as serving as a important source of employment for almost 1.5 billion people. Livestock animal division incorporates creature cultivation, dairy and fisheries part are extensive significant segments. It assumes a significant function in public economy and social financial advancement of the nation.

As livestock animals come and go in their daily routine, they are counted as they come in close enough proximity to the counter. Instead of driving into a field and interactively counting a herd. If the number displayed is less than the known headcount the rancher or herdsman could either do a manual headcount or check the counter again later in the day and transmission of a headcount from a remotely operation system, are possible. The most common and difficult job of a rancher or herdsman is counting their head to derive count so they can determine animal availability. It's a task that should be done once a day. It takes a very large amount of the rancher's or herdsman's time and thus an automated solution is proposed. The system is designed to be as very simple to use and as affordable to purchase as possible in an effort to make this invention a basic and viable tool of any rancher or herdsman with a medium-sized herd on up to the largest.

\subsection{Motivation}

Farmer need to be updated on new technologies and management practices of livestock production on regular basis. One of the common problems faced by the commercial livestock farms is taking the headcount of the livestock animals. The same problem is faced by small ranch owners. To solve this issue, we are developing a system that is used to detect a particular animal and take the headcount accordingly. The system is created in such a way that it is simple to use and affordable.

\subsection{Aim and Objectives}

The main focus is to develop an automated model which has the capability of identifying the livestock image or video is given as a test image from the dataset. It can classify the Livestock animals without human intervention. The system can classify Livestock animals with high accuracy. The main objective of our project is...

- To capture the image of the livestock 's using a camera. 
- To identify the livestock animal and classifying them using image processing with Convolutional Neural Network.

- Detect the type of the intruder if any.

\section{Literature Survey}

Stringa [1] The primary objective of such surveillance system is to alert the operator when a dangerous situation is encountered over the camera and the operator has to record this incident and this is only possible by the insertion of image processing capabilities added to the system which also allows to capture complex events or the detection of longterm changes and patterns moving in the scene near detected changes. These complex events can be used both for online and for off-line retrieval of potentially dangerous situation together with their causes. The system employees object classification for retrieving objects. The recognition feature mostly works with the geometry of the objects for classifying them. For this to work the objects should not change their present position for long time the concept is that there is a moving person in proximity of the position of an abandoned object, in the instant in which the object is left. More precisely, taking into account that meaningful changes are detected by the change detection module after 16 frames starting from the moment $\mathrm{X}$ in which probably the object is abandoned, it is interesting to study the movement in a temporal slot The movement is studied within a set of pixels belonging to a region surrounding the abandoned object. Jason Wang [2] We propose two different approaches to data augmentation. The first approach is generate augmented data before training the classifier. For instance, we will apply GANs and basic transformations to create a larger dataset. All images are fed into the net at training time and at test time, only the original images are used to validate. The second approach attempts to learn augmentation through a prepended neural net. At training time, this neural net takes in two random images from the training set and outputs a singleimage so that this image matches either in style or in context with a given image from the training set. This output, which represents an augmented image produced by the network, is fed into the second classifying network along with the original training data. $\mathrm{R}$ Cutler [3] describes that the main contribution of this work is the introduction of novel techniques to robustly detect and analyses periodic motion. The demonstration of this techniques is done with video of the quality typically found in both ground and airborne surveillance systems. The interest is in utilizing the symmetries of motion displayed in nature, which we use for object Identification. We also provide various other novel applications of periodic motion, all related to automating a surveillance system.

\section{Methodology}

\subsection{Data Preprocessing}

The data is collected from the Kaggle website which contains 2000 of photos of each livestock animal like sheep. After the data is collected, the data is processed for augmentation. Here we use the method called data augmentation that expand and increase the size of a training dataset by making modified versions of images in the dataset, the capability to fit models using image data augmentation by the Image Data Generator class is provided by The Keras deep learning library.It is in common knowledge that the more data an $\mathrm{CNN}$ algorithm has access to, the more effective it can be. Data augmentation is another method we used to reduce overfitting on models, where we increased the amount of training data pictures using information only in our training data.

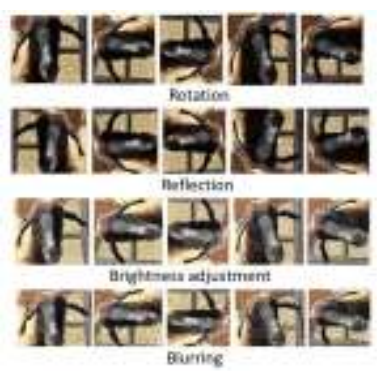

Fig. 1. Augmented image sample. After the data is augmented the images are preprocessed this includes Mean subtraction and scaling is done to keep all images of data in same size.

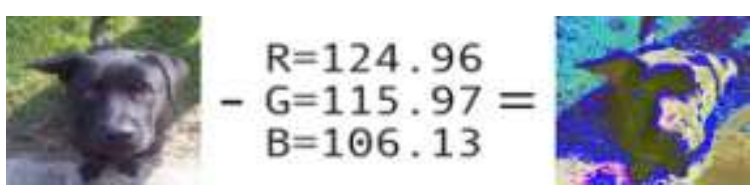

Fig. 2. Mean subtraction

\subsection{CNN architecture and Training}

The input layer, output layer and with different hidden layers make up the convolutional neural network.Some of these hidden layers are convolutional, using a mathematical model to pass on results to successive layers.

- Along with the raw pixel values of the pictures and also with the 3 color channels R, G, B will be provided as Input. 
- The neurons that are connected to local regions in the input and their output is calculated by Convolution layer from calculating each dot product between their weights and region they are connected to in the input volume.

- The element-wise activation function is applied by RELU layer and the size of the volume is unaffected.

- The downsampling operation along the spatial dimensions (width, height) is executed by POOL layer which results in volume such as [16x16x12].

\section{Convolutional Layer-}

The extracting of features from an given image is done by convolution layerThe relationship between pixels is maintained by Convolution by learning image features using a small matrix of input data. Inputs like image matrix and filter could be used for the mathematical operation. The operations such as edge detection, blur and sharpening will be achieved by using various filters from Convolution layer.

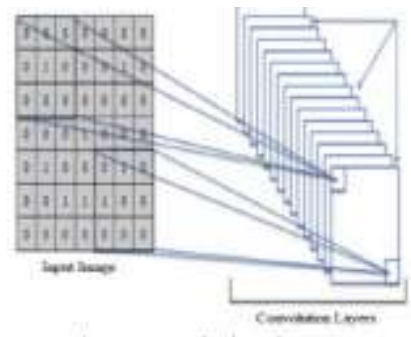

Fig. 3: ConvolutionalLayer

\section{Pooling-}

The number of parametersare reduced by Pooling layer when the images are large. Max pooling will take the largest element from the rectified feature map. By reducing the dimensionality, the input representation will be down-sampled and this is the main objective of polling layer.
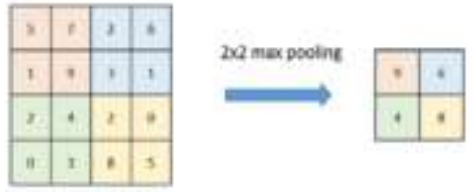

Fig. 4: Pooling Layer

\section{Flattening-}

Flattening is the process in which there will be converting all the resultant 2 dimensional arrays into a single long continuous linear vector. The one large feature vector is created by flattening all the structures ofthe output from the convolutional layer, which will be used by the heavy layer for the ultimate classification.
ISSN 2455-6378

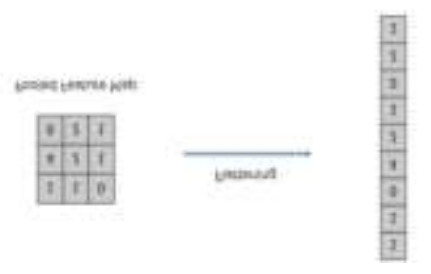

Fig. 5: Flattening Layer

\section{Fully connected-}

The Fully Connected Layers are the ones which are the hidden layers inside a Convolutional Neural Network.The Fully Connected Layersare a type of hidden layers which are used within the Convolutional Neural Network. The more accurate output is predicted by combining the features into more attributes.

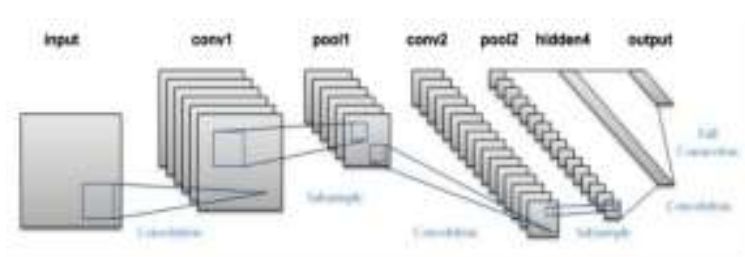

Fig. 6: Fully connected layer

After the CNN model is created. The collected data set is divided into Train and Test set. After we will randomly choose $80 \%$ of the data as Training dataset and the remaining $20 \%$ to be the Validation set, the model is then continuously trained and validated on these different sets.

\subsection{Animal Classification}

The live video is captured in the ranch and it is converted to frames (series of images). Then the images are looped through the detections and secondary frames of varying height and width are drawn over the resized image for detection to trained $\mathrm{CNN}$ model. We are extracting confidence and then comparing the confidence threshold. If the confidence is a minimum threshold, we proceed to draw secondary frames with the probability of detection. Then the images inside the frame are converted to greyscale forcomparison.After the detection of animal is confirmed the result is displayed along with the count.

\section{Implementation}

\subsection{Hardware Implementation}

The video of the livestock animals is collected in real-time by using camera for testing data which are used during the image processing method. 


\subsection{Software Implementation}

The Camera is connected with raspberry pi and the CNN model is implemented in the raspberry pi. The livestock images will be given as input for the application for animal classification and counting them. The training data is collected from kaglle website for every livestock animal and the CNN model is trained with training dataset and $20 \%$ of data is kept for validation. The functions such as index Image, image Set and retrieve Image are from the program.Index Image is used alongside with the retrieve Image function to search for images. The camera will capture the image and this image is given as input for the system to classify. The two main arguments that the retrieve function takes isa input image and the image saved in the database.The result is the indices according to images within image Index that are visually identical to the query image. The image id output includes the indices in ranked order, from the most match to least identical match. The $0-1$ is the range of threshold value. If the threshold value is near to 0 , then the image is not matched. If the threshold value is near to 1 , them the image is matched with the image in database and the animal is classified. If the identified animal name does not matches with the ranchers livestock animals then it is identified as an intruder animal. If the threshold value is in the range of 0.2 to 0.8 , then the image is compared with that of the stored image.

\section{Results}

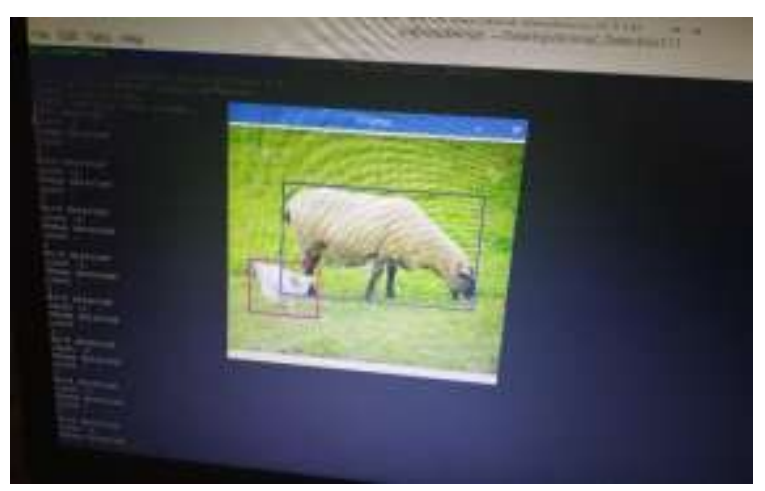

Fig. 7: Detection of Sheep and Counting

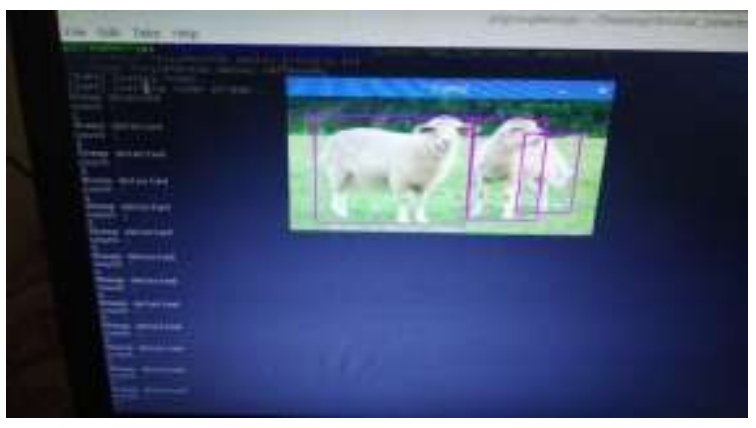

Fig. 8: Detection of Sheep and Counting

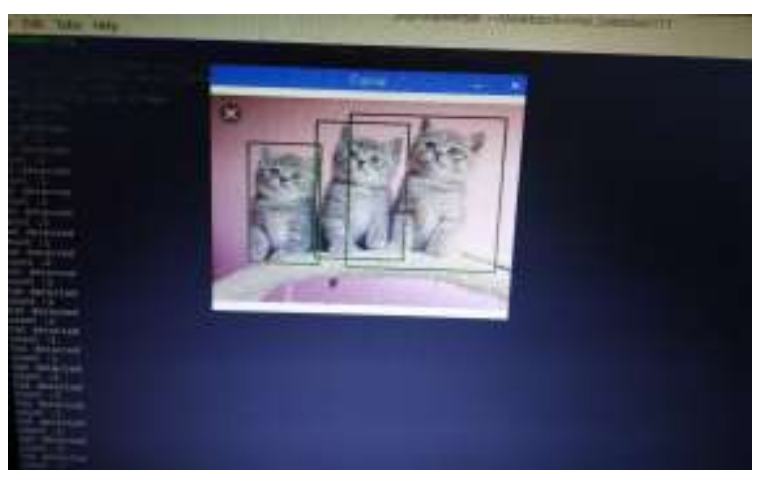

Fig. 9: Cats Detection with count

\section{Conclusions}

This project implements Convolutional Neural Network (CNN) deep learning algorithm to classify and counting livestock animals. The project classifies livestock animals efficiently with a89\% of accuracy, and also the detected animals are counted this helps to keep count of them and also detect any intruder animal in the ranch. This project is affordable by both small scale and large-scale livestock ranchers and this is achieved by using raspberry pi. In this project first it captures video and video is converted to frames and then the frames are converted into grey scale image after that the CNN model classify and counts the animal in the image. The presentapplications like bar code scanning and manually counting of livestock is not beneficial as it consumes a lot of time and the error margin becomes high so to overcome such hurdles. This project is designed to be a real-time system that solves the problem and improves efficiency, accuracy and it is affordable.

\section{References}

[1] .E. Stringa and C.S. Regazzoni, Contentbased retrieval and real time detection from video sequences acquired by surveillance 
systems, Proceedings 1998 International Conference on Image Processing. ICIP98 (Cat. No.98CB36269), 7-7 Oct. 1998

[2] The Effectiveness of Data Augmentation in Image Classification using Deep Learning, Jason Wang, Luis Perez December-2017

[3] R Cutler and L.S Davis, Robust real-time periodic motion detection, analysis, and applications, IEEE Transactions on Pattern

\section{ISSN 2455-6378}

Analysis and Machine Intelligence, Volume: 22, Issue: 8 , Aug 2000.

[4] Mayur J Charadva, Ramesh V Sejeal, Dr. Nisha P Sarwade, A Study of Motion Detection Method for Smart Home System, IJIRAE, Volume 1, Issue 5, June 2014.

[5] Outdoor wildlife motion triggered camera recreational camera traps for wildlife management and conservation research. (Shafika, Suhaimi,2015) 